\title{
Uni- and multidirectional wear resistance of different crosslinking degrees in UHMWPE for artificial joints
}

\author{
V. A. González-Mora ${ }^{1}$, M. Hoffmann ${ }^{1}$, R. Stroosnijder ${ }^{1}$, E. Espinar ${ }^{2}$, J. M. Llamas ${ }^{2}$, \\ M. Fernández-Fairén ${ }^{3}$, F. J. Gil ${ }^{3}$ \\ ${ }^{1}$ Institute for Health and Consumer Protection, Joint Research Centre, European Commission, Ispra, Italy; \\ ${ }^{2}$ Grupo de investigación en Ortodoncia, Facultad de Odontología, Universidad de Sevilla, Seville, Spain; \\ ${ }^{3}$ CREB, Department Ciencia de Materiales e Ingeniería Metalúrgica, ETSEIB, Universidad Politécnica de Cataluña, Barcelona, \\ Spain. \\ Email: francesc.xavier.gil@upc.edu
}

Received 20 December 2010; revised 17 February 2011; accepted 24 February 2011.

\begin{abstract}
The aim of this work was to study the effect of UHMWPE crosslinking on wear performance. Differently treated UHMWPEs were studied by means of unidirectional and multidirectional wear tests. Unidirectional tests simulate total knee replacement and multidirectional tests simulate total hip replacement movements. The samples tested were observed by optical and scanning electron microscopy in order to determine wear mechanisms that explain the different results obtained in the uni- and multidirectional wear tests performed.
\end{abstract}

Keywords: Artificial Joints; Wear; Polymeric Biomaterials

\section{INTRODUCTION}

When natural joints have to be replaced with artificial materials, their tribological properties change as a result of the inability of the materials to produce a permanent, artificial lubricating film. Therefore, the materials used for articulating components in an artificial joint are inevitably subject to wear. Furthermore, there is no ideal bearing material that currently fulfils all the requirements of arthroplasty design [1,2]. It is therefore important to ensure that wear is minimised to avoid possible aseptic loosening following osteolysis due to particleinitiated foreign body reaction [3-5].

The articulating surfaces in total joint replacement are recognised as major sources of wear debris generation. Other implant surfaces, specifically fixation surfaces, may release additional wear debris during the in vivo function. Thus, the origin of wear particles can be divided into the prosthesis-bone interface and the prosthesis-prosthesis interface, which can be designed to be articulating or non-articulating. The amount of wear debris from the former interface is low. This may be acceptable, but only if the debris does not migrate to other interfaces where it could contribute to third-body wear [6].

The lack of an adequate standard hampers the comparison of studies carried out by various laboratories and the progress made in understanding the wear phenomena that occurs in total joint replacements for classifying the various UHMWPEs studied [7-10]. More must be done than simply reviewing or improving the existing standards. Furthermore, there is a clear need to develop a new standard for screening wear tests based on the latest findings, such as multidirectional motion or lubricant composition.

\section{MATERIALS AND METHODS}

A pin-on-disk (POD) wear-test machine is a common wear-test method that has been widely used to evaluate the wear of polymers in biotribology. In a POD test, the polymer, usually in the form of a pin, slides over the surface of a rotating disk. Two basic configurations may be employed: the pin is loaded along the main axis of the disk either perpendicular or parallel to its axis of rotation. Hence, the contact area is either on the edge (horizontal POD configuration) or on the face (vertical POD configuration) of the disk. According to this definition, the wear-test method proposed here was a horizontal POD (hereinafter simply called POD I).

In the POD test device, a vertically positioned wheel or ring rubs against a polyethylene pin below it. Figure 1(a) shows a diagram of the loading/motion configuration. The contact geometry at the start of the test is non-conformal, that is, in line contact. Therefore, these wear-test devices would be more representative of a knee-wear device than of a hip-wear device, for which 


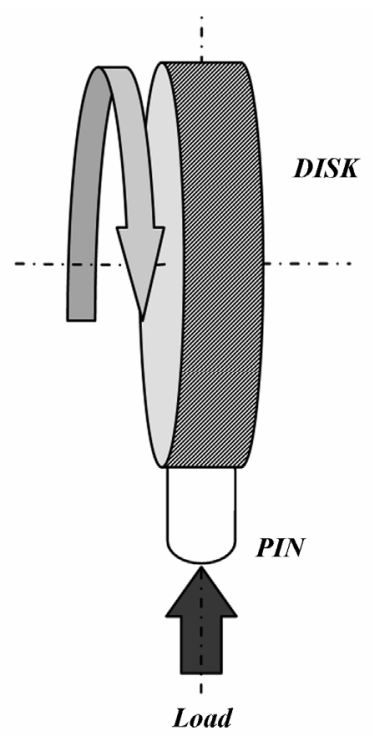

(a)

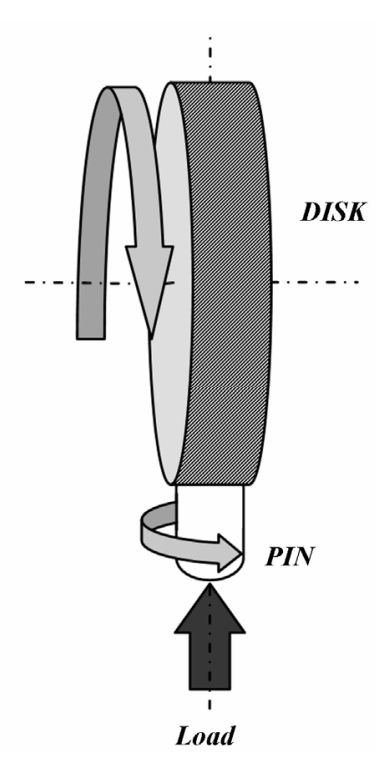

(b)

Figure 1. Motion/loading configuration of the POD wear test machines. (a) Unidirectional. (b) Multidirectional.

the contact geometry is conformal (also known as congruent).

There is no standard regarding this type of wear device nor are there any similar studies in the literature. Thus, a comparison and analysis of the test conditions proposed cannot be made. Due to its configuration, the closest available standard would be the ASTM G137-97 standard, which has identical contact geometry and is also intended for classifying the resistance of plastic materials in sliding wear. However, this standard does not cover biotribological conditions.

The multidirectional POD (POD II) wear-test method is based on the former unidirectional POD (POD I) wear-test method. However, in the POD II test method, the pin also rotates (see Figure 1(b)) and, as consequence of the rotation of the pin, this device displays a biaxial (i.e. multidirectional) motion. In the literature, no wear device similar to this can be found, which makes it a unique screening wear-test device. In theory, the tasks undertaken using this wear-test method are the same as those undertaken using the former POD I. Additionally, the effect of the motion type in the wear resistance of the UHMWPE may be studied by means of the POD II tests.

The test using the POD test method was performed as follows. The disk rotated continuously against a UHMWPE pin placed beneath it. A load of 150 N (15 kg) was applied to the pins, which gave a maximum Hertzian contact pressure $\left(p_{0}\right)$ of $\sim 5 \mathrm{MPa}$. The relative surface velocity was $100 \mathrm{rpm}$ for the disk; in the case of the multidirectional test, the pin velocity was $99 \mathrm{rpm}$. The disk had a frequency of $1 \mathrm{~Hz}$. The wear of the UHMWPE pins was mainly determined by profilometric measurements. Weight loss measurements were also performed after the completion of the test. The test length was 34 $\mathrm{km}$.

Distilled water was added to the test lubricant during the test to compensate for water evaporation. A solution consisting of bovine serum and distilled water was used as the test lubricant, which had a total protein concentration of $30 \mathrm{mg} / \mathrm{ml}$. The serum was purchased from Sigma-Aldrich (calf serum, bovine donor; product no. C9676). The fluid adsorption rate of the UHMWPE pins was determined using an additional control pin, which was loaded in exactly the same way as the UHMWPE pins in the RPOF machine, but no motion was applied. The cleaning and drying of the UHMWPE pins was performed according to the ASTM 1715 standard. Weighing was carried out using a Mettler Toledo AT261-DeltaRange $^{\circledR}$ microbalance with an accuracy of $\pm 10 \mu \mathrm{g}$.

The disks were made of forged CoCrMo alloy (purchased from Firth Rixson Superalloys Ltd., Derbyshire, England). The disks were $88 \mathrm{~mm}$ in diameter and $10 \mathrm{~mm}$ thick. The roughness of the disks was determined by laser profilometer, which yielded a value of $R_{a}=0.01$ $\mu \mathrm{m}$. The pins were manufactured from a bar of UHMWPE GUR1050, which was $13 \mathrm{~mm}$ in length and $9 \mathrm{~mm}$ in diameter. Four different treatments on the UHMWPE material were studied.

- Non-treated

- Crosslinked I ( $\gamma$-sterilised + stabilised I) with the following treatment:

- By irradiation with $100 \mathrm{kGy}$ of gamma ray in air (sterilisation)

- By the McKellop heat treatment at $155^{\circ} \mathrm{C}$ for 
72 hours in nitrogen (stabilisation)

- Crosslinked II ( $\gamma$-sterilised + stabilised II) with the following treatment:

- By irradiation with $100 \mathrm{kGy}$ of gamma ray in air (sterilisation)

- By heat treatment under water at $130^{\circ} \mathrm{C}$ in $\mathrm{H}_{2} \mathrm{O}$ for 72 hours (stabilisation)

- Sterilised with a standard 25 kGy (2.5 Mrad) of gamma radiation in air

For each UHMWPE material, three samples were tested. A total of 12 wear tests were performed. Test conditions and materials are summarised in Table 1.

\section{EXPERIMENTAL RESULTS AND DICUSSION}

The wear results, given in volumetric wear units $\left(\mathrm{mm}^{3}\right)$, are calculated from the average weight loss (mg) recorded for three specimens of each UHMWPE material. A higher weight loss of the UHMWPE material represents greater wear in the sample. A graph of the wear results is shown in Figure 2.

\subsection{Unidirectional Wear Test}

For the unidirectional test, the results showed higher wear for the irradiated and crosslinked UHMWPEs (XLPEs) than for the unirradiated UHMWPE material. The UHMWPE wear obtained for the irradiated materials and XLPEs were in the same order, yielding a 1.5 to 1.8 fold increase with respect to the unirradiated UHMWPE. A high reproducibility of the wear tests was achieved, in which the low standard deviation in the weight loss of the specimens did not ever exceed $7 \%$. The results show that the difference between crosslinked materials is not statistically significant $(p<0.05)$, and that the two crosslinking treatments seem to have a similar effect on the wear resistance of the UHMWPE. The results also demonstrate that the unidirectional motion applied is unable to differentiate between irradiated

Table 1. Test conditions of the POD wear tests.

\begin{tabular}{cc}
\hline Test parameter & Value \\
\hline Contact geometry & Cylinder-on-flat (non-conformal) \\
Frequency & $1 \mathrm{~Hz}$ \\
Relative surface velocity & $100 \mathrm{rpm}$ \\
Contact area & Line \\
Load applied & $\sim 150 \mathrm{~N}(15 \mathrm{~kg})$ \\
Contact stresses & $5 \mathrm{MPa}$ \\
Test length & $34 \mathrm{Km}(123000 \mathrm{disk}$ rotations $)$ \\
Lubricant & $30 \mathrm{mg} / \mathrm{ml}$ initial protein content \\
Temperature & Room \\
Counterface component & CoCrMo alloy \\
UHMWPE component & Non-treated \\
(GUR 1050) & Crosslinked I $(\gamma$-sterilised + stabilised I) \\
\end{tabular}

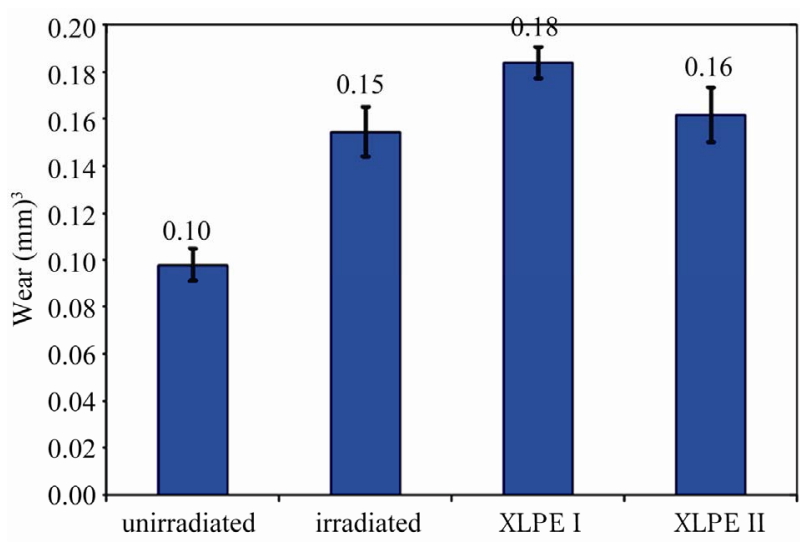

Figure 2. Average wear of the UHMWPE pins in the unidirectional POD wear tests.

materials (irradiated UHMWPEs and XLPEs), even if they are quite different. Due to the four-fold irradiation dose (100 kGy), XLPEs have a higher degree of crosslinking compared with UHMWPEs that are irradiated with $25 \mathrm{kGy}$. Despite this difference in the degree of crosslinking, when a unidirectional sliding motion is applied they display a similar degree of resistance. The significance of the results obtained by employing unidirectional motion is questionable, because wear tests using this kind of motion only seem to be able to detect differences in the wear behaviour of unirradiated and irradiated UHMWPEs, while differences between different degrees of crosslinking cannot be detected.

Questions may arise regarding the influence of load on UHMWPE wear, for example, if a difference in the wear of XLPEs were encountered whenever higher loads were applied. In our opinion, the use of higher loads may result in higher UHMWPE wear for all the materials studied here, but the classification of materials would remain unchanged. Studies [11,12] focusing on the effect of load on UHMWPE wear have concluded that this is not a key parameter when an attempt is made to classify the UHMWPE.

The surface of the pins was observed by means of optical microscopy (OM), as can be seen in Figure 3(a). In Figure 3(b), the surface resulting from the machining process shows the marks left behind by the machining tool's cutting action. The main features are the scratches caused by the action of the disk sliding over the pin's surface, which leaves a typical unidirectional lay structure, in line with the sliding direction of the disk on the pin's surface (see Figure 3(c)). To the naked eye, the wear zone appears to be polished. Furthermore, besides the scratches mentioned above, the continuous sliding of the disk on the pin's surface in the wear zone formed a ripple-like microstructure or lay of the fibres that make up the UHMWPE. The ripples are perpendicular to the 


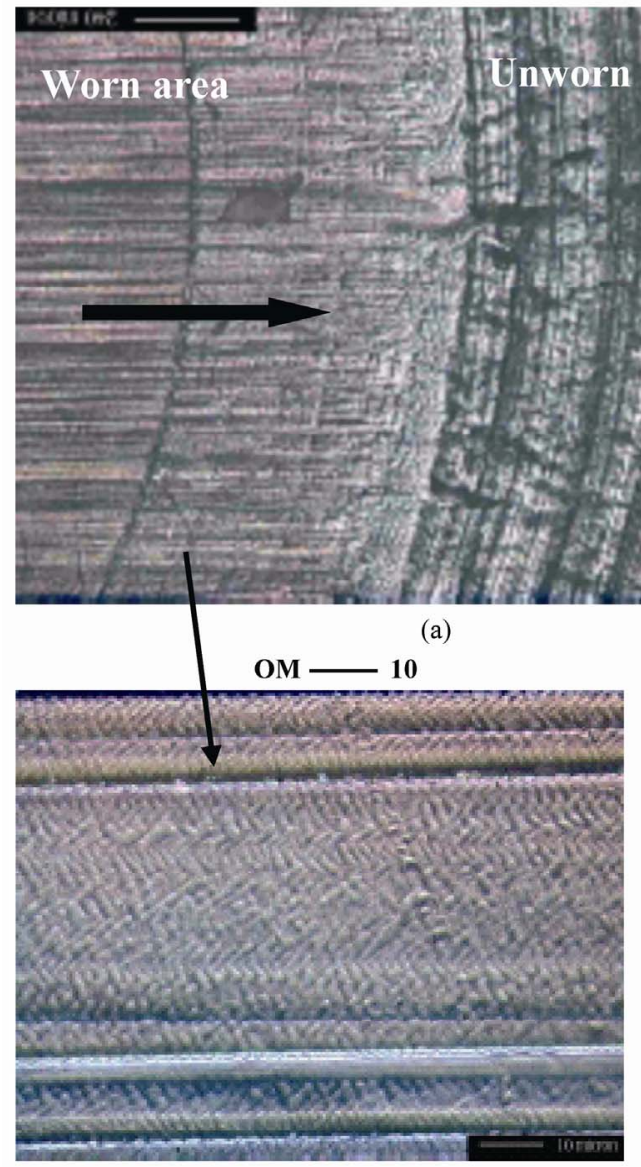

(c)

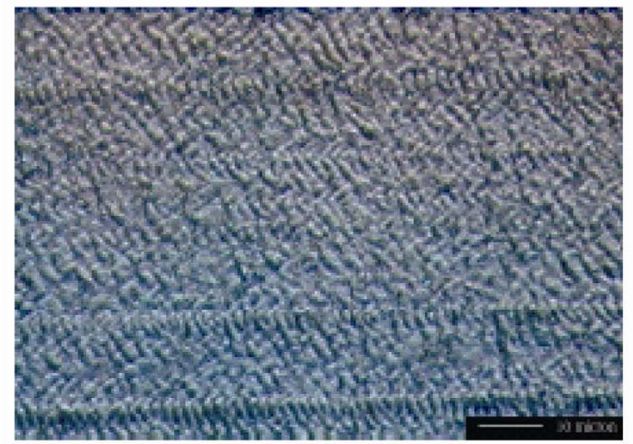

(d)

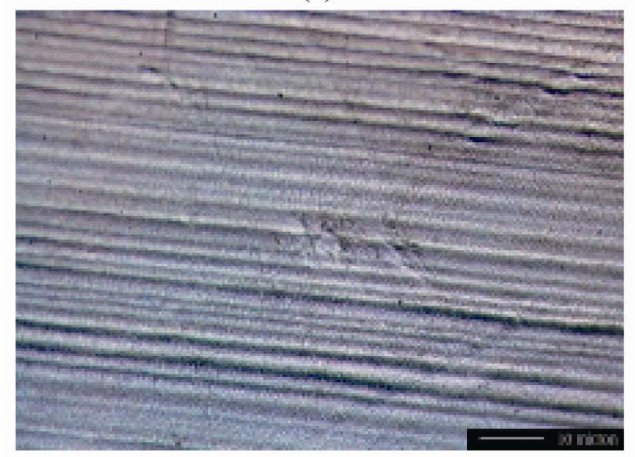

(e)

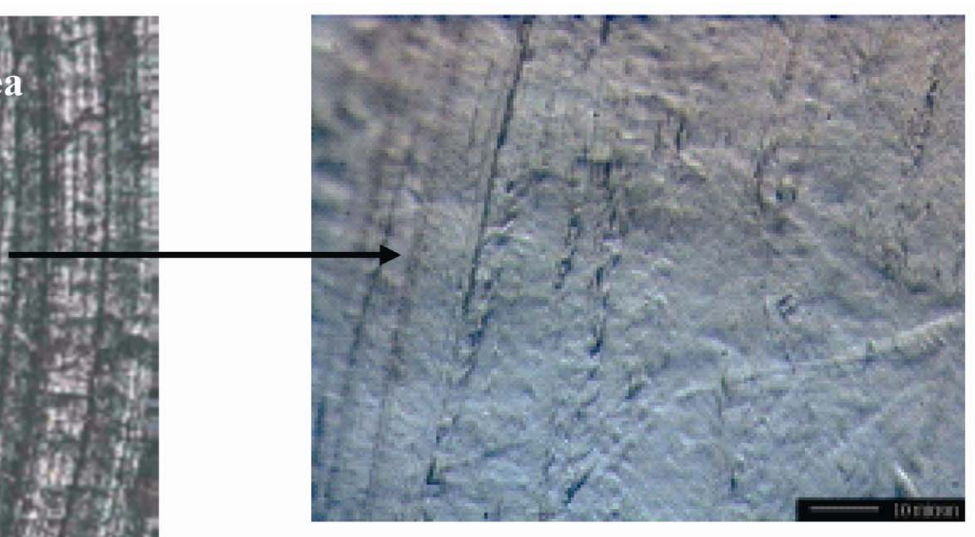

(b)

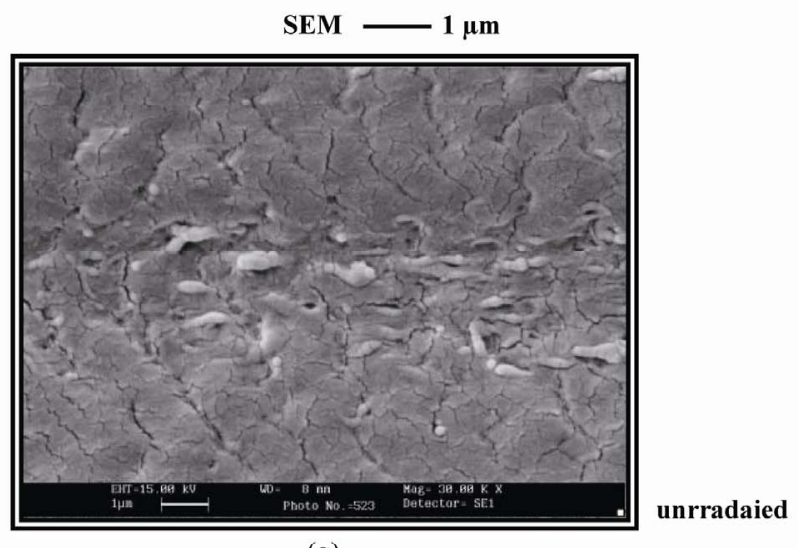

(g)

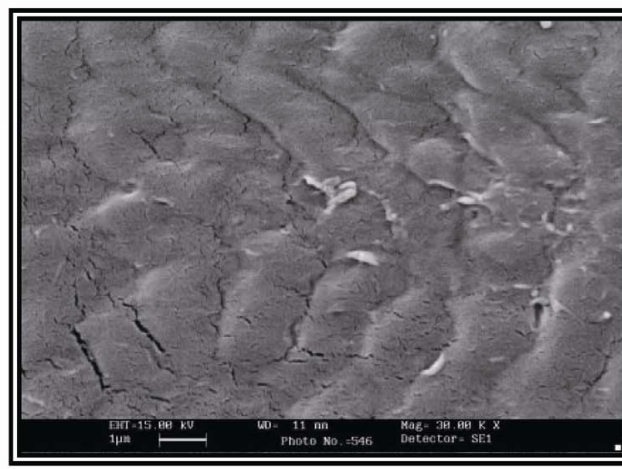

Irradiated (25 kGy)

(h)

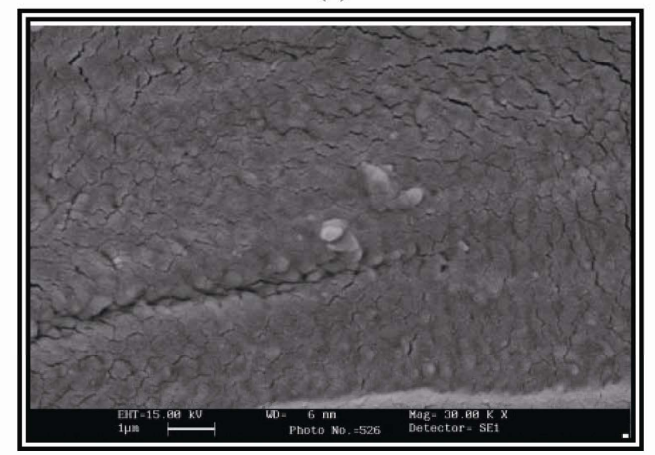

(i)

XLPE I (100 kGy) 


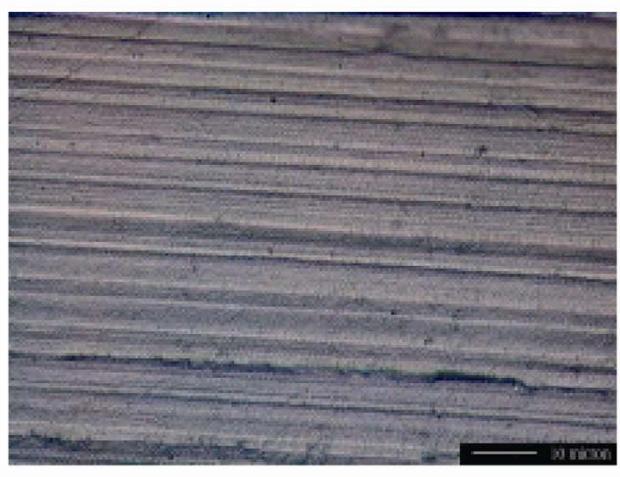

(f)

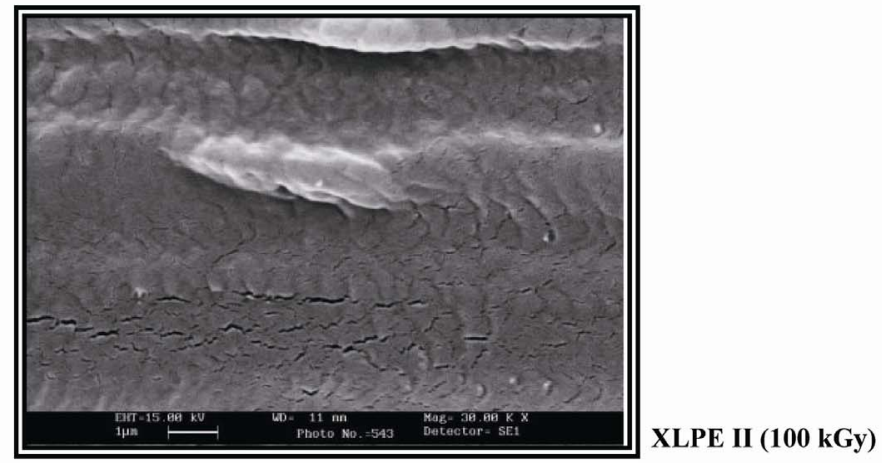

(j)

Figure 3. (a) Image of the unirradiated UHMWPE pin after the unidirectional wear test. The black arrow shows the direction of sliding. (b) Detail of the machined surface. (c) Detail of the worn surface of unirradiated UHMWPE. Note also the scratches parallel to the sliding direction. (d) Detail of the worn surface of irradiated UHMWPE. (e) Detail of the worn surface of XLPE I. (f) Detail of the worn surface of XLPE II. (g) to (j) Images obtained by SEM.

sliding direction. It could be concluded that the microstructure of the UHMWPE fibres clearly falls into alignment with the sliding direction.

Optical micrographs of the worn surfaces revealed the ripple-like structure on all the pins studied (Figure $3(\mathbf{d})$ ). However, the size of the ripples was different if the pin was made of an XLPE material (Figures 3(e) and 3(f)). The ripple-like structure is clearly smaller compared with the microstructure found in the unirradiated or irradiated UHMWPEs. The ripples on XLPEs are smaller because their fibres are smaller in comparison to noncrosslinked UHMWPEs. This is a consequence of the heat treatment that XLPEs undergo after irradiationperformed at $155^{\circ} \mathrm{C}$ in the case of these materials-which acts as a remelting process for the UHMWPE fibres. Another feature of the worn surfaces is that the irradiated UHMWPEs and XLPEs have a greater number of scars than the unirradiated UHMWPEs, and that the scars are shallower. Both the morphology of the ripples and the scratches displayed a lower degree of deformation in the irradiated UHMWPEs and XLPEs, due to the irradiation-induced crosslinking process.

The observation by means of SEM focused on the formation of UHMWPE particles that detach from the pin surface, which gives rise to wear debris. The scanning electron micrographs of the unirradiated (Figure 3(g)), irradiated (Figure 3(h)), XLPE I (Figure 3(i)) and XLPE II (Figure 3(j)) materials are shown below.

The SEM observations (Figures $3(\mathrm{~g})$ to $\mathbf{3}(\mathrm{j})$ ) show that the UHMWPE pins exhibit a cracked surface texture. This texture shows up as micro-cracks, which are present in every direction but most frequently occur between ripples, thus highlighting the ripple-like microstructure of the UHMWPE when it is observed under an optical microscope. These micro-cracks are, however, a result of gold sputtering, which is necessary to bring about conductivity on the UHMWPE surface. They do not show up as micro-cracks on the UHMWPE surface. This effect could not be avoided even if the sputtering periods were very short.

Besides the ripple-like microstructure, other features can be observed, such as the particle formation in the form of fibrils on the worn surfaces. The study of the particle formation is essential to understanding the wear processes that occur, as a hypothesis has been advanced that wear particles may be liberated from the articulating surface after the cyclic accumulation of a critical amount of plastic strain.

There is a greater degree of fibril formation in the unirradiated material than in the irradiated material, but less so in the case of XLPEs. For non-XLPEs, fibrils are placed parallel to the sliding direction and may extend over several ripples. In the case of XLPEs, rounded particles smaller than those that occur in non-XLPEs are formed rather than fibrils. The particle formation in the XLPEs once again corroborates the fact that there tends to be less deformation in the XLPEs in comparison with irradiated and unirradiated UHMWPEs. In Figure 3(j), the concentration of plastic strain on the polyethylene surface in the XLPEs can be observed.

With regard to the results for weight loss, the particle formation in the XLPEs should have been higher than in the non-XLPEs, since UHMWPE wear was also higher. At this point, it is worth remembering the phenomenon of irrecoverable, permanent strain resulting from mechanical loading, which is termed plasticity and holds clues as to the wear and mechanical loading history of UHMWPEs. The higher production of wear debris in the XLPEs may be explained by the fact that the particles that form on the XLPEs immediately become detached. This is due to the fact that they are able to bear a lower strain concentration (5.6) than non-XLPEs particles, 
which coincides with the appearance of rounded and smaller XLPE particles. However, non-XLPEs have a higher deformation capacity (or plasticity), allowing particles to retain a higher accumulation of plastic strains and resulting in less particle detachment and, consequently, less weight loss. This coincides with the known fact that the molecular orientation of UHMWPE occurs under unidirectional sliding conditions, including anisotropy in the UHMWPE and orientation hardening in the sliding direction [11]. In conclusion, UHMWPEs with a greater capacity to deform locally (i.e. with greater plasticity) present higher wear resistance and less weight loss under unidirectional conditions. For the same reasons, there is less wear in unirradiated UHMWPE than in irradiated UHMWPE, as this and other studies have shown, since the latter possesses lower plasticity due to sterilisation (irradiation process).

\subsection{Multidirectional Wear Test}

The wear results of the UHMWPE samples (pins) obtained using the POD II (multidirectional) test method are shown in Figure 4.

The wear results show a higher wear rate for the irradiated and unirradiated UHMWPE materials compared to the XLPEs. That is, the unirradiated UHMWPE materials and the irradiated UHMWPE materials in particular perform worse than the crosslinked materials. In fact, the wear observed in the irradiated and unirradiated UHMWPEs was 3.5 and 5 times higher, respectively, than that in XLPE II. Moreover, wear in the irradiated material was 1.43 -fold higher than that in the unirradiated UHMWPE. XLPE I and II displayed similar wear resistance, although the latter showed slightly lower wear than XPLE I, which is statistically significant $(p<0.01)$. A high reproducibility of the wear tests was achieved for all the materials tested, which is expressed by the low standard deviations (SD $<5 \%$ ).

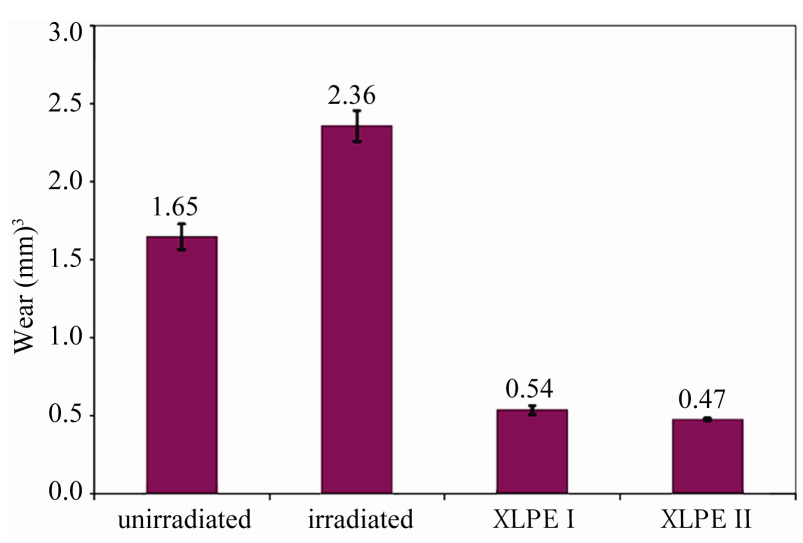

Figure 4. Average wear of the UHMWPE pins in the multidirectional POD wear tests.
Contrary to the POD I test method, in which multidirectional sliding conditions were employed, the POD II test method showed that multidirectional motion is able to differentiate between irradiated materials in terms of their wear rates, i.e. between XLPEs and irradiated UHMWPEs. The results also demonstrated that under multidirectional sliding conditions XLPE materials improve the tribological resistance of UHMWPEs, which exhibit 4.4. to 5 times less wear compared with the standard irradiated UHMWPE component. For example, assuming that an acetabular cup made of standard irradiated UHMWPE has a common mean lifetime of 10 to 15 years, the higher wear resistance of XLPEs could increase the mean lifetime by 45 to 66 years.

This comparison between uni- and multidirectional results leads to two conclusions:

a) Multidirectional sliding motions cause greater UHMWPE wear than unidirectional sliding motions.

b) The classification of the materials tested shows an inverse trend under unidirectional and multidirectional sliding motions.

The two classes of wear results-the inverse classification and the order of magnitude of UHMWPE wearcoincide with the results obtained in studies found in the literature. This supports the POD wear-test method applied in this work as a suitable screening wear test for the evaluation of UHMWPE wear.

The multidirectional wear results performed on the POD machine also show that the XLPEs are an optimal material for acetabular bearing components in total hip replacements, since UHMWPE wear can be significantly reduced with respect to the standard irradiated (25 kGy) UHMWPE. The results under unidirectional sliding conditions show, however, that the XLPEs are subject to more wear compared with the unirradiated UHMWPE. Therefore, in situations in which the unidirectional sliding motion is the main type of motion between the articulating components, as encountered in most current total knee replacement designs, XLPEs should not be used. It is now recognised that the different kinematics in the hip and knee can lead to a different wear mechanism surface and wear rate of the UHMWPE component [12]. For total hip replacements, it is now widely accepted that wear is related to the mechanical response of UHMWPE materials under multidirectional conditions. In total hip replacements, variation in the direction of the velocity vector leads to cross shearing on the strainhardened polyethylene and accelerates wear. However, for total knee replacements, the major contribution made to sliding motion seems to be unidirectional motion, depending on the prosthesis design, and thus the response of UHMWPE under unidirectional conditions seems to be the most significant. Retrieved total knee components present scratches on the UHMWPE com- 
ponent that are predominantly parallel to the wear sliding direction [6,7]. Thus, in total knee replacements with a unidirectional motion, the wear may be lower due to the orientation and strain hardening of the UHMWPE in the direction of motion. Two factors affecting UHMWPE wear and behaviour that have not yet been satisfactorily addressed must be taken into account: the high variabi- lity in knee designs and the much higher stress contacts in comparison with hip designs.

The optical microscopies at low magnification are presented in Figures 5(a) to 5(d). They show that the unirradiated and irradiated UHMWPEs exhibit greater wear damage when they are compared to XLPEs, which are consistent with the weight loss results. It should be
$\mathrm{OM}-10 \mu \mathrm{m}$

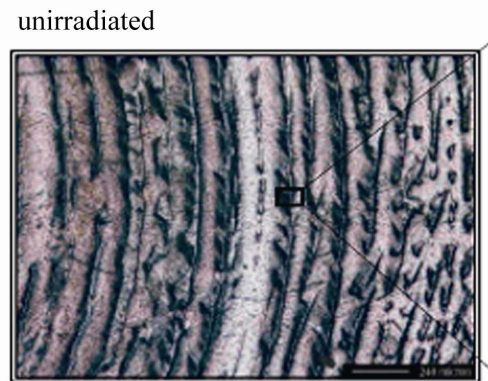

(a)

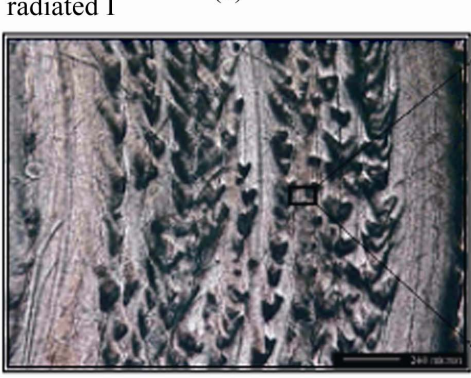

(b)

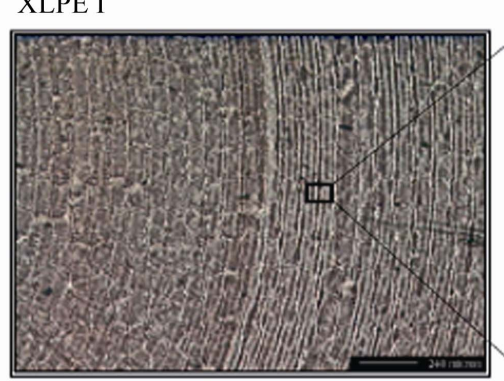

(c)

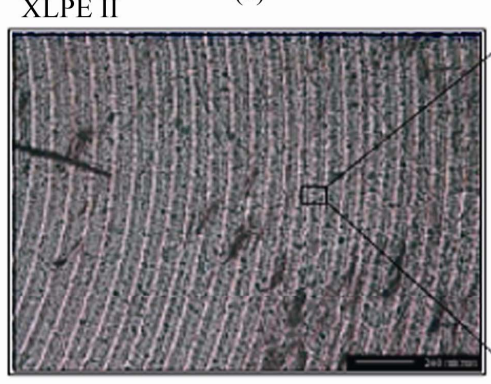

(d)

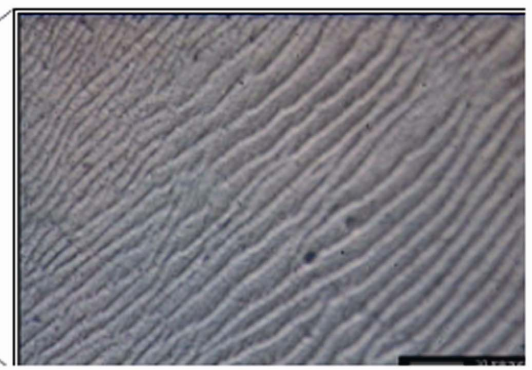

(e)

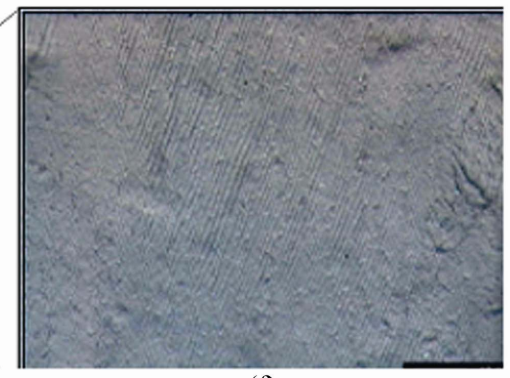

(f)

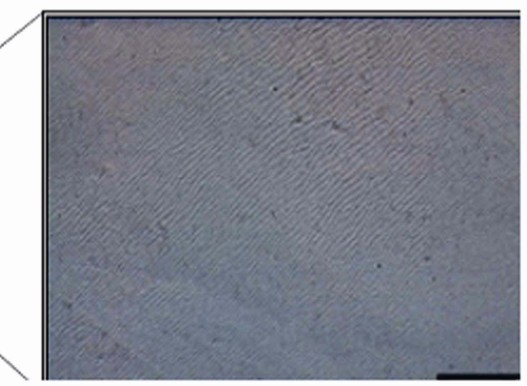

(g)

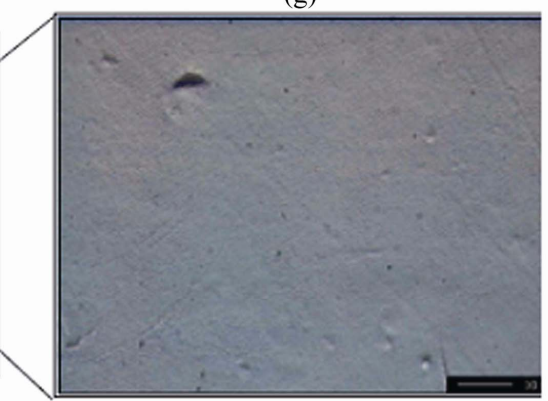

(h)

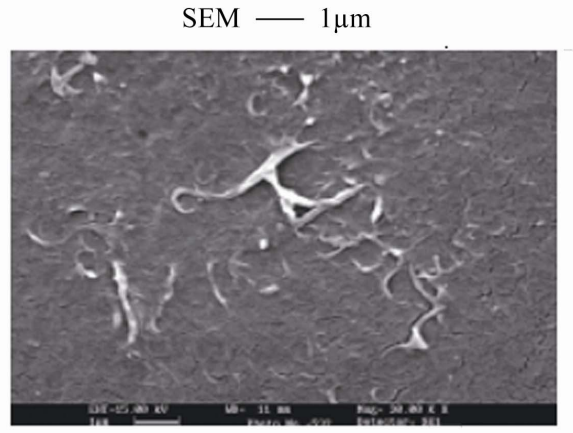

(i)

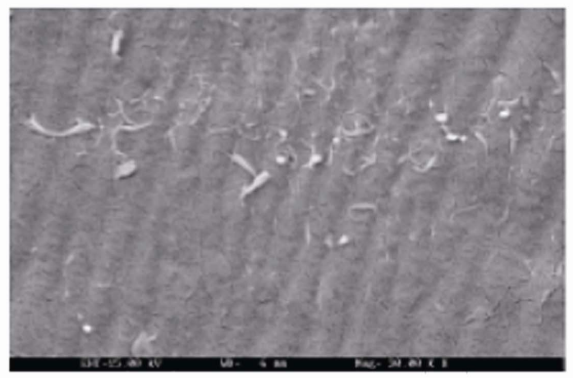

(j)

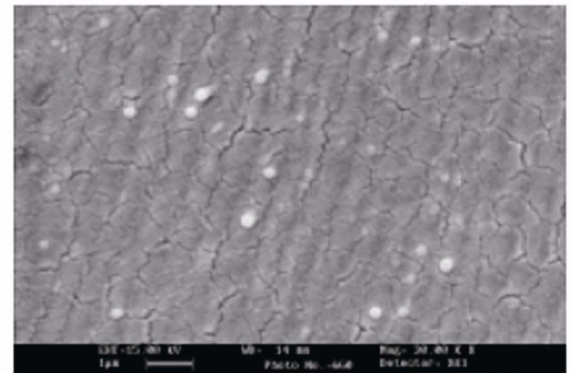

(k)

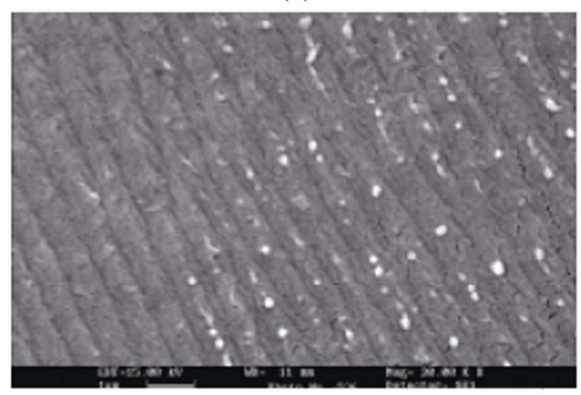

(1)

Figure 5. (a) Image of the surface of the unirradiated UHMWPE pin after the multidirectional wear test, (b) for irradiated UHMWPE, (c) for XLPE I, (d) XLPE II, (e) to (h) Details of the microstructure observed by optical microscope. (i) to (l) Details of the microstructure observed by SEM. 

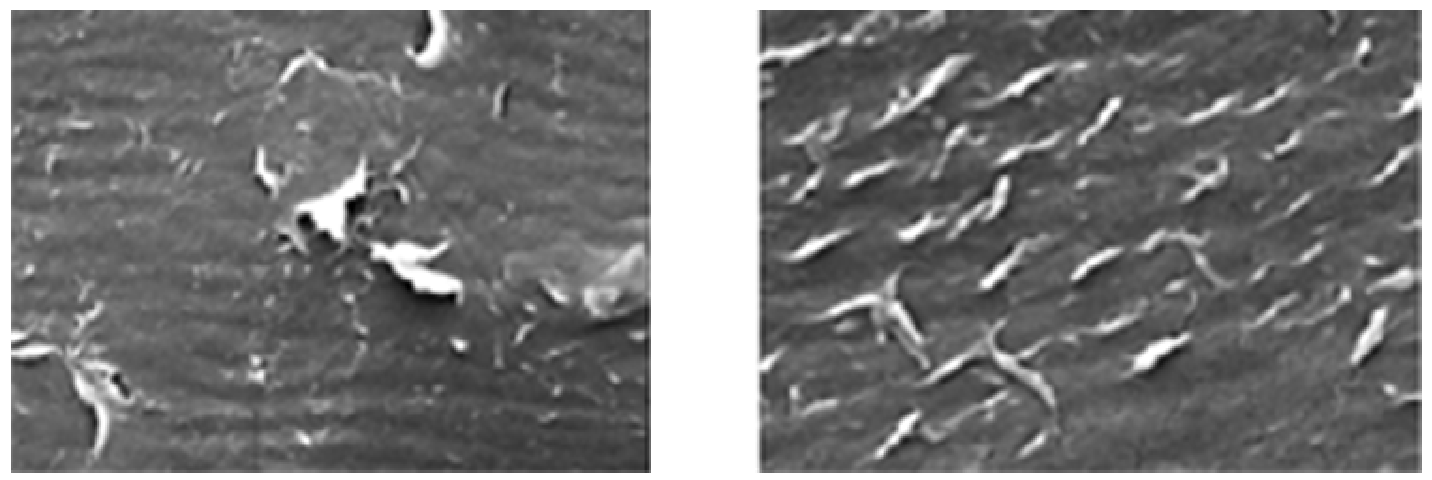

Figure 6. Region of the unirradiated UHMWPE pin: SEM micrographs after multidirectional wear test.

noted that parallel scratches found on the pin surfaces in the unidirectional tests (POD I) are not present here and only multidirectional shallow scratches can be seen.

Optical microscopy images at higher magnification were taken at the midpoint of the pin's diameter (see Figures 5(e) to 5(h)). The central plateau was not considered because the optical microscope was unable to observe any features other than the flatness of this area. The figures reveal a similar ripple-like microstructure for all the pins studied. In this case, the ripples are less aligned than those found in the unidirectional wear tests. Again, the microstructure of the ripples is more evident in the unirradiated and irradiated UHMWPEs than in the XLPEs, which reinforces the theory that the XLPEs have lower plasticity, as discussed in the results on unidirectional wear. The ripples are perpendicular to the sliding direction, which under the multidirectional sliding condition yielded results in which the ripples were radially oriented, as if the sliding directions were from the centre of the pin to its edge.

The SEM observation was focused on the formation of UHMWPE particles that detach from the pin surface to produce wear debris. The scanning electron micrographs of the unirradiated (Figure 5(i)), irradiated (Figure 5(j)) XPLE I (Figure 5(k)) and XLPE II (Figure 5(l)) materials are shown below.

The ripple-like microstructure identified by optical microscopy is present in the area outside the central plateau, both for the unirradiated and irradiated UHMWPEs. The central plateau of the pin shows an extremely homogeneous and flat microstructure with no visible ripples. However, there is particle and fibril formation on the central plateau. The formation of fibrils was also observed on the rest of the worn surface. As can be observed in Figure 6, all the fibrils are oriented along the sliding direction and are perpendicular to the ripple-like microstructure (radially from the pin's centre to the pin's edge), and these fibrils can extend over several ripples. Fibril formation is more marked for the unirradiated than for the irradiated material, followed by the crosslinked materials.

For the XLPEs, the homogeneous ripple-like microstructure can be seen all over the pin's surface. Fibril formation is less marked for the XLPEs than for the unirradiated and irradiated UHMWPEs. Compared with the non-crosslinked UHMWPEs, XLPEs show much less particle formation and the fibrils are smaller. As far as the unidirectional tests are concerned, the fibrils are much rounder and smaller, which under the SEM appear as white particles forming on the ripples of the microstructure. As explained for the unidirectional wear tests, this particle formation in XLPEs is caused by their lower plasticity.

Based on the size of the fibrils forming on both noncrosslinked UHMWPEs and XLPEs, the size of the particles forming the UHMWPE debris can be estimated. Figures 5(i) to 5(l) and Figure 6 show that the size of the UHMWPE particles that detach from the worn surfaces ranges from a submicron to more than one micron. It has been found that in total hip replacements most UHMWPE particles are less than one micron in length [13]. The findings of studies of wear particles retrieved from periprosthetic tissues and analyses of worn polyethylene surfaces are consistent with an average particle size in the 0.5 micrometer diameter range [3,7].

\section{REFERENCES}

[1] Miller, D.A., Herrington, S.M., Higgins, J.C. and Schroeder, D.W. (1995) UHMWPE polyethylene in total joint replacement: History and current technology. In: Wise, D.L., et al. Eds., Encyclopaedic Handbook of Biomaterials and Bioengineering, Part B: Applications, Marcel Dekker, Inc., New York, 665-688.

[2] Greer, K.W., Hamilton, J.V. and Cheal, E.J. (1995) Polyethylene wear in orthopaedics. In: Wise, D.L., et al. Eds., Encyclopaedic Handbook of Biomaterials and Bioengineering, Part B: Applications, Marcel Dekker, Inc., New York, 613-638. 
[3] Murray, D. and Rushton, N. (1990) Macrophages stimulate bone resorption when they phagocytose particles. Journal of Bone and Joint Surgery, 72-B, 988-992.

[4] Howie, D. and McGee, M. (1996) Wear and osteolysis in relation to prostheses design and materials. Medical Applications of Titanium and Its Alloys (ASTM STP 1272).

[5] McGee, M., Howie, D., Neale, S., Haynes, D. and Pearcy, M. (1997) The role of polyethylene wear in joint failure. Proceedings of the Institution of Mechanical EngineersPart H, 211, 65-72. doi:10.1243/0954411971534692

[6] Green, T., Fisher, J., Stone, M., Wroblewski, B. and Ingham, E. (1998) Polyethylene particles of a "critical size" are necessary for the induction of cytokines by macrophages in vitro, Biomaterials, 19, 2297-2302. doi:10.1016/S0142-9612(98)00140-9

[7] Greer, K.W., Hamilton, J.V. and Cheal, E.J. (1995) Polyethylene wear in orthopaedics. In: Wise, D.L., et al. Eds., Encyclopaedic Handbook of Biomaterials and Bioengineering, Part B: Applications, Marcel Dekker, Inc., New York, 613-638.

[8] Plitz, W. and Walter, A. (1994) Tribological aspects of metal/polymer couplings. In: Buchhorn, G. and Willert,
H.G., Eds., Technical Principles, Design and Safety of Joint Implants, Hogrefe \& Huber Publishers, Cambridge, 82-89.

[9] Algan, S.M. and Horowitz, S.M. (1995) Biology of aseptic loosening of the cemented arthroplasty. In: Wise, D.L., et al. Eds., Encyclopaedic Handbook of Biomaterials and Bioengineering, Part B: Applications, Marcel Dekker, Inc., New York, 773-797.

[10] Viceconti, M., Cavallotti, G., Andrisano, A. and Toni, A. (1996) Discussion on the design of a hip joint simulator. Medical Engineering \& Physics, 18, 234-240. doi:10.1016/1350-4533(95)00026-7

[11] Ben Abdallah, A. and Treheux, D. (1991) Friction and wear of utrahigh molecular weight polyethylene against various new ceramics. Wear, 142, 43-56. doi:10.1016/0043-1648(91)90151-J

[12] Suh, N., Mosleh, M. and Arinez, J. (1998) Tribology of polyethylene homocomposites. Wear, 214, 231-236. doi:10.1016/S0043-1648(97)00233-0

[13] Streicher, M. (1995) Tribology of artificial joints. In: Morscher, E., Ed., Endoprosthetics, Springer Verlag, Berlin. 